\title{
Hydromagnetic Wind Model for the Broad-Line Region of NGC 5548
}

\author{
M. C. Bottorff, K.T. Korista, and I. Shlosman \\ Department of Physics and Astronomy, University of Kentucky, \\ Lexington, $K Y 40506$, USA \\ R. D. Blandford \\ Theoretical Astrophysics, 130-33, Caltech, Pasadena, CA 91125, USA
}

\begin{abstract}
A hydromagnetic wind model from a dusty molecular disk (Emmering, Blandford, \& Shlosman 1992) was applied to the BLR in NGC 5548 and produced synthetic C IV line profiles as a function of time. The model C IV profiles are compared to data from the $1993 \mathrm{IUE} / \mathrm{HST}$ campaign and properties of the the BLR of NGC 5548 are inferred.
\end{abstract}

\section{The Model}

Gas clouds are centrifugally flung off a molecular and dusty disk along magnetic field lines forming a wind. The rising clouds become exposed to the central continuum and emit line radiation.

The model line core $\left( \pm 3000 \mathrm{~km} \mathrm{~s}^{-1}\right)$ and mid-wings $\left( \pm 3000-6000 \mathrm{~km} \mathrm{~s}^{-1}\right)$ reflect the kinematics of the CIV-emitting gas and the cloud properties. The $3 \mathrm{D}$ velocity field of the wind is a combination of rotation and outflow. Clouds are subject to magnetic confinement, are optically thick, and CIV photons are emitted anisotropically. The surface emissivity of clouds is calculated by using CLOUDY for an amalgam of optically thick clouds exposed to the best fit continuum shape for NGC 5548 (Walter et al. 1994). Optical depth effects (cloud obscuration) are accounted for. A static narrow-line component is added to the core of the C IV profile.

The line wings ( $\left.\pm 6000-9000 \mathrm{~km} \mathrm{~s}^{-1}\right)$ are formed through local scattering of the line core photons by hot $\left(\sim 10^{6} \mathrm{~K}\right)$ electrons surrounding the evaporating clouds. The model is driven by the observed 1993 continuum at $1460 \AA$. The synthetic line profiles are shown in Fig. 1 superimposed on the data profiles.

\section{Conclusions}

We reach the following conclusions (Bottorff et al. 1996): (1) The BLR in NGC 5548 is modeled by a hydromagnetic outflow of optically thick clouds from an opaque accretion disk around $\mathrm{a} \sim 3 \times 10^{7} M_{\odot}$ black hole. (2) The BLR has a disklike shape extending $\pm 30^{\circ}$ from the equatorial plane. (3) The observer's aspect angle is $40^{\circ}$ with respect to the symmetry axis of the disk. (4) The C IV-emitting 


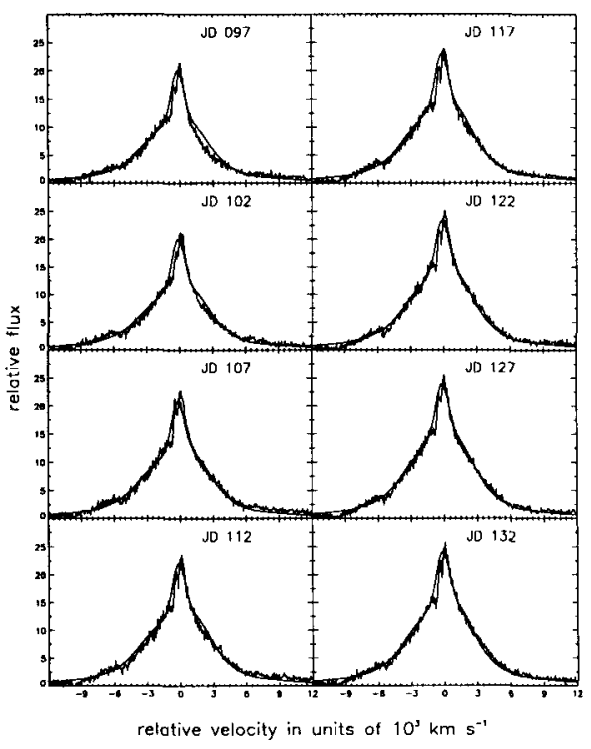

Figure 1. Comparison of model (smoother lines) and predicted line profiles for $\mathrm{C}$ IV in NGC 5548 during the IUE/HST campaign. The Julian dates (minus $2,449,000$ ) are shown in the upper right corners. The vertical axis is given in relative intensity units. The horizontal axis gives the Doppler shift in $\mathrm{km} \mathrm{s}^{-1}$ with respect to the line center (negative velocities are blueshifted). From Bottorff et al. (1996)

region lies between 1 and 24 light days from the source of ionizing photons. (5) The outer C IV boundary is determined by cloud-cloud obscuration of the central continuum. Low-ionization lines, like Mg II, are expected to originate farther out, where the central UV-soft-X-ray continuum is attenuated. (6) The model reproduces successfully the observed CIV line profiles and the CIV variability in NGC 5548; the integrated line flux lags the continuum by $\sim 8$ days. (7) We predict a differential response across the CIV profile with the red mid-wing $\left(3000-6000 \mathrm{~km} \mathrm{~s}^{-1}\right)$ leading the response. The red side leads the blue side and the wings lead the core. (8) The outer wind has similar spectral characteristics to a molecular torus, although its dynamics are fundamentally different.

\section{References}

Bottorff, M., Korista, K., Shlosman, I. \& Blandford, R. 1996, ApJ, submitted. Emmering, R. T., Blandford, R. D. \& Shlosman, I. 1992, ApJ, 385, 460.

Korista, K. T., et al. 1995, ApJS, 97, 285.

Walter, R., et al. 1994, A\&A, 285, 119. 